\title{
PRODUÇÃO E RELAÇÃO RÁQUIS/CACHO DA BANANEIRA 'NANICÃO’ EM DIFERENTES DENSIDADES E ARRANJOS DE PLANTIO'
}

\author{
RICARDO ALFREDO KLUGE², JOÃO ALEXIO SCARPARE FILHO², RICARDO VICTÓRIA FILHO² \\ e ANGELO PEDRO JACOMINO²
}

\begin{abstract}
RESUMO - Objetivou-se avaliar a influência de diferentes densidades e arranjos de plantio sobre os componentes de produção e a relação ráquis/cacho de bananeiras 'Nanicão', estabelecidas em Piracicaba, SP. Foram estudadas quatro densidades: 1.333, 1.666, 2.222 e 3.333 plantas ha $^{-1}$, e dois arranjos de plantio: retângulo e triângulo (quincôncio). Independentemente do arranjo utilizado, o aumento da densidade elevou a produção de frutos de 37,24 para 75,83 $\mathrm{t} \mathrm{ha}^{-1}$ e reduziu a massa do cacho de 30,30 para $24,79 \mathrm{~kg}$, por causa da redução na massa do fruto. A porcentagem da massa da ráquis em relação à massa do cacho permaneceu em torno de $8 \%$, independentemente da densidade e arranjo. Na maior densidade (3.333 plantas ha $\mathrm{a}^{-1}$ ) podem ser exportadas do bananal até $6,80 \mathrm{t} \mathrm{ha}^{-1}$ de ráquis, material que pode ser aproveitado como matéria-prima para a fabricação de papel.
\end{abstract}

Termos para indexação: Musa, população de plantas, distribuição da população, espaçamento, rendimento, inflorescências.

\section{YIELD AND BUNCH STEM/BUNCH RATIO OF ‘NANICÃO’ BANANAS AT DIFFERENT DENSITIES AND ARRANGEMENTS}

\begin{abstract}
This research was carried out with the objective to evaluate the effects of different densities and plant arrangement on components of yield and bunch stem/bunch ratio of 'Nanicão' banana established in Piracicaba, SP. Four densities (1,333, 1,666, 2,222 and 3,333 plants ha $\left.{ }^{-1}\right)$ and two arrangements (rectangle and triangle) were studied. Independent of arrangement the increase of density raised fruit yield of 37.24 to $75.83 \mathrm{tha}^{-1}$. However bunch mass was reduced of 30.30 to $24.79 \mathrm{~kg}$ due to reduced fruit mass. The bunch stem/bunch ratio was $8 \%$ independent of density and arrangement. At density of 3,333 plants ha- ${ }^{-1}$ can be exported $6.80 \mathrm{t} \mathrm{ha}^{-1}$ of bunch stem of plantation and these component of yield can be used as raw material to manufacture paper.
\end{abstract}

Index terms: Musa, plant population, stand establishment, spacing, yield, inflorescences.

\section{INTRODUÇÃO}

Dados referentes ao ano de 1998 apontam que o Brasil é o terceiro maior produtor de bananas, com total aproximado de 5,5 milhões de toneladas ano ${ }^{-1}$, depois da Índia e Equador, que produzem 10,2 e 7,5 milhões de toneladas ano $^{-1}$, respectivamente (FAO, 1999).

\footnotetext{
${ }^{1}$ Aceito para publicação em 20 de dezembro de 1999.

${ }^{2}$ Eng. Agrôn., Dr., Dep. de Produção Vegetal, Escola Superior de Agricultura Luiz de Queiroz (ESALQ), Caixa Postal 9, CEP 13418-900 Piracicaba, SP. Bolsista do CNPq. E-mail: rakluge@carpa.ciagri.usp.br, jascarpa@carpa.ciagri.usp.br rvictori@carpa.ciagri.usp.br, jacomino@carpa.ciagri.usp.br
}

$\mathrm{O}$ adensamento de plantio tem sido visto como uma estratégia para aumentar a produtividade, pois conduz, normalmente, a um melhor aproveitamento do solo, mão-de-obra e insumos e a elevadas produções por área.

A densidade ótima de plantio na cultura da banana é derivada de uma integração complexa de muitos fatores, tais como: cultivar, fertilidade do solo, tipo de muda, seleção de seguidores (rebentos), nível de tecnologia aplicada, controle de plantas daninhas, velocidade do vento, topografia, aspectos econômicos, entre outros (Simmonds, 1982; Robinson \& Nel, 1988; Soto Ballestero et al., 1992). Quando as diferenças climáticas são adicionadas a esses fatores, torna-se óbvio que as respostas das plantas às dife- 
rentes densidades de plantio podem diferir substancialmente de uma região a outra. Tem sido verificado que a densidade de plantas interfere na massa de cacho e na produtividade dos bananais. De maneira geral, o aumento na densidade diminui a massa do cacho, em virtude da redução no número de pencas e frutos (Mattos et al., 1970; Santos, 1977; Gomes et al., 1984; Robinson \& Nel, 1989). Contudo, o adensamento eleva a produtividade, principalmente nos primeiros ciclos (Daniells et al., 1985; Lichtemberg et al., 1996).

A distribuição ou arranjo das plantas na área de plantio também constitui um fator de influência sobre a produtividade, pois os diferentes sistemas de espaçamento alteram a eficiência na captação de luz pelas folhas das plantas bem como afetam a exploração do solo (Pereira, 1989; Robinson et al., 1989). Os arranjos de plantio mais comumente usados são o quadrado e o retângulo, que geralmente apresentam baixa eficiência no aproveitamento do terreno e da luz (Soto Ballestero et al., 1992), enquanto outros arranjos, como o triângulo, são pouco estudados em bananicultura.

A cultura da banana produz uma quantidade grande de resíduos orgânicos, que incluem o pseudocaule, folhas, engaço (pedúnculo) e ráquis. Moreira (1987) estimou que um bananal conduzido de maneira convencional pode fornecer até $200 \mathrm{t} \mathrm{ha}^{-1} \mathrm{ano}^{-1}$ de restos de cultura. Os restos vegetais, deixados após a colheita, permanecem no bananal como forma de disponibilizar matéria orgânica às plantas que estão em desenvolvimento (Gallo et al.,1972; Purseglove, 1972). Normalmente, o pseudocaule é mantido o mais comprido possível, até 45-50 dias após o corte do cacho, para favorecer o desenvolvimento dos rebentos (Moreira, 1987), enquanto as folhas velhas, ao cobrir o solo, podem auxiliar no controle de plantas daninhas. Adicionalmente, essas folhas são incorporadas ao solo, fornecendo nutrientes.

Com a adoção de tecnologia de colheita mais apropriada, existe no Brasil a tendência de substituir o encaixotamento de bananas no campo pelo transporte dos cachos inteiros até o galpão de embalagem, para posterior tratamento e embalagem. Isso fará com que a ráquis e o engaço sejam retirados do bananal, tornando-se pouco aproveitados caso não haja retorno dos materiais à plantação. As partes do cacho, entretanto, podem ser aproveitadas como matéria-prima na obtenção de fibra para a produção de papel. Segundo Torres (1981), a fibra da ráquis apresenta $11,73 \%$ de lignina e 53,5\% de alfa-celulose, suplantando, em celulose, a fibra do bagaço de cana-de-açúcar (Saccharum spp.). Sua maior resistência e flexibilidade, de acordo com o autor, faz desse material uma fonte apropriada para a produção de papel. Blanco Rojas (1996), trabalhando com beneficiamento e polpação da ráquis da bananeira 'Nanicão', verificou que, no Estado de São Paulo, cerca de $2.000 \mathrm{t} \mathrm{ano}^{-1}$ de pasta celulósica poderiam ser obtidas a partir desse material.

Os dados referentes à massa da ráquis e seu porcentual em relação à massa do cacho são escassos. Isso faz com que a produtividade estimada de um bananal seja calculada, invariavelmente, com base na massa total do cacho. Daniells et al. (1985) determinaram que a ráquis da bananeira 'Williams' (Musa AAA subgrupo Cavendish) representa aproximadamente $9 \%$ da massa total do cacho. Blanco Rojas (1996) observou em Registro, Vale do Ribeira, SP, que a massa da ráquis da bananeira 'Nanicão' variou de 1,28 a 2,88 kg, com média de $1,8 \mathrm{~kg}$.

A interferência da densidade de bananais sobre a massa da ráquis e seu porcentual em relação à massa do cacho não tem sido estudada.

O objetivo deste trabalho foi verificar o efeito de diferentes densidades de bananeiras, estabelecidas em dois arranjos de plantio, sobre os componentes exportáveis de produção e sobre o porcentual da massa da ráquis em relação à massa do cacho.

\section{MATERIAL E MÉTODOS}

$\mathrm{O}$ experimento foi conduzido na área experimental de banana do Departamento de Produção Vegetal da Escola Superior de Agricultura “Luiz de Queiroz”, Piracicaba, SP.

O clima de Piracicaba, conforme a classificação de Köppen, é do tipo Cwa: tropical de altitude, com três meses mais secos (junho/julho/agosto), chuvas de verão e seca no inverno. A temperatura média do mês mais quente é maior que $22^{\circ} \mathrm{C}$, e do mês mais frio não é inferior a $16^{\circ} \mathrm{C}$, com média de $21,1^{\circ} \mathrm{C}$; precipitação média de $1.253 \mathrm{~mm}^{2} \mathrm{ano}^{-1}$; ventos predominantes $1^{\underline{a}}$ este e $2^{2}$ sudoeste, com velocidade média de $2,2 \mathrm{~m} \mathrm{~s}^{-1}$; umidade relativa do ar de $74 \%$ e 
insolação média mensal de 201,5 horas. A altitude de Piracicaba é de $546 \mathrm{~m}$.

O solo da área experimental foi classificado como Terra Roxa Estruturada eutrófica A moderado textura argilosa sobre muita argilosa, correspondendo ao Kandudalfic Eutrudox (Vidal-Torrado \& Sparovek, 1993).

Foi acompanhado o terceiro ciclo de bananeiras cultivar Nanicão (Musa AAA subgrupo Cavendish) plantadas em 24 de janeiro de 1994. Foram utilizadas mudas do tipo "chifrão" (brotação lateral, com folhas lanceoladas e emitindo folhas normais, com peso médio de $2,5 \mathrm{~kg}$ ) e o bananal foi conduzido com três plantas por touceira (planta matriz, primeiro rebento e segundo rebento). A colheita do referido ciclo ocorreu entre os meses de março e junho de 1997.

Durante o ciclo da cultura foram realizadas as práticas de controle de plantas daninhas, capinas, desbaste de rebentos, retirada de folhas velhas, adubações, retirada do coração e controle de pragas e doenças, conforme recomendações de Moreira (1987). O coração foi eliminado 10 dias após a abertura da última penca, deixando-se a ráquis masculina com aproximadamente $12 \mathrm{~cm}$. A última penca foi mantida no cacho.

Os tratamentos consistiram do uso de diferentes densidades e arranjos de plantio. Os arranjos utilizados foram retângulo e triângulo (quincôncio). Cada arranjo de plantas apresentou quatro densidades: 1.333, 1.666, 2.222 e 3.333 plantas $\mathrm{ha}^{-1}$, representando um total de oito tratamentos. No arranjo em retângulo, as dimensões utilizadas foram 3,0 x 2,5 m, 3,0 x 2,0 m, 3,0 x 1,5 m e 3,0 x 1,0 m. Para o arranjo em triângulo, as dimensões (base $\mathrm{x}$ altura) foram: 2,8 x 1,4 m, 2,6 x 1,3 m; 2,4 x 1,2 m e 2,0 x 1,0 m. Em cada vértice do retângulo e do triângulo foi colocada uma planta.

O delineamento experimental adotado foi o de blocos casualizados em esquema fatorial $4 \times 2$ (quatro densidades e dois arranjos), com três repetições. Cada parcela, incluindo a bordadura, foi constituída de 30 a 45 plantas, sendo consideradas 10 plantas úteis, o que totalizou 240 plantas avaliadas. Os dados coletados foram submetidos à análise de variância (teste F) e, em caso de significância, as médias foram comparadas pelo teste de Tukey a 5\% de probabilidade.

As seguintes variáveis foram avaliadas: a) massa do cacho $(\mathrm{kg})$ : cada cacho foi pesado logo após a colheita; b) massa da ráquis ( $\mathrm{kg}$ ): considerou-se como ráquis o conjunto entre o engaço (porção do cacho que inicia no ponto de fixação da última folha e termina na inserção da primeira penca) e a ráquis propriamente dita (eixo onde se inserem as inflorescências, iniciando no ponto de inserção da primeira penca e terminando na inflorescência masculina ou coração); c) massa de pencas por cacho (kg): calculada subtraindo-se a massa da ráquis da massa do cacho; d) porcentagem da massa da ráquis (MR) em relação à massa do cacho (MC) (\% MR/MC); e) número de frutos por cacho; f) massa média do fruto (g): obtida pela divisão da massa de pencas por cacho pelo número de frutos presentes em cada cacho; g) número de pencas por cacho; h) produção estimada de cachos $\left(\mathrm{t} \mathrm{ha}^{-1}\right)$ : calculada multiplicando-se a massa do cacho pelo número de plantas por hectare; i) produção estimada de frutos $\left(\mathrm{t} \mathrm{ha}^{-1}\right)$ : calculada multiplicando-se a massa de pencas pelo número de plantas por hectare; j) produção estimada de ráquis $\left(\mathrm{t} \mathrm{ha}^{-1}\right)$ : calculada mediante a multiplicação da massa da ráquis pelo número de plantas por hectare.

\section{RESULTADOS E DISCUSSÃO}

A análise da variância mostrou que não houve efeito do arranjo de plantio em nenhuma das variáveis, o mesmo ocorrendo na interação entre densidade e arranjo. Isso indica que há pouca influência da disposição das plantas sobre os componentes de produção da cultivar em questão, e que o efeito das densidades, aos níveis estudados, independe do arranjo das plantas no terreno, ao menos no terceiro ciclo.

O aumento da densidade de plantas tendeu a diminuir a massa do cacho (Tabela 1). A maior densidade (3.333 plantas ha-1) reduziu em 15 a 20\% a massa do cacho em comparação às demais densidades. Esses resultados concordam com os encontrados por outros pesquisadores (Mattos et al., 1970; Santos, 1977; Gomes et al., 1984; Robinson \& Nel, 1989). A diminuição na massa do cacho provocada pelo aumento da densidade deve-se, provavelmente, à maior competição interplantas por água e nutrientes, e pior aproveitamento da luminosidade, que ocorrem sob altas populações de plantas (Janick, 1968; Robinson et al., 1989).

Embora não tenha havido diferença significativa, a massa da ráquis tendeu a ser menor com o aumento da densidade (Tabela 2), acompanhando a redução da massa do cacho. Assim, na maior densidade a massa da ráquis foi aproximadamente $15 \%$ menor do que nas demais densidades. A média geral da massa da ráquis observada no experimento foi de $2,30 \mathrm{~kg}$, superior à média de $1,88 \mathrm{~kg}$ observada por Blanco Rojas (1996), no município de Registro. 
A porcentagem média MR/MC observada no presente trabalho $(8,08 \%)$ foi inferior à obtida por Daniells et al. (1985) na Austrália, cujo valor médio foi de $9 \%$. É provável que outros fatores, além da densidade, como cultivar, clima e solo, estejam envolvidos nessa relação. Embora o teste $\mathrm{F}$ da análise da variância não tenha acusado efeito dos tratamentos, houve uma tendência de a porcentagem da relação MR/MC diminuir à medida que a densidade foi reduzida, passando de $8,20 \%$, em média, na densidade 3.333 plantas ha-1, para $7,78 \%$ na densidade 1.333 plantas ha(Tabela 1). Isso indica que, com a redução na compe-

TABELA 1. Massa do cacho, ráquis e pencas, e relação entre massa da ráquis e massa do cacho (MR/MC) da bananeira 'Nanicão' em diferentes densidades e arranjos. Piracicaba, SP, 1997.

\begin{tabular}{|c|c|c|c|}
\hline \multirow{2}{*}{$\begin{array}{c}\text { Densidade } \\
\left(\text { plantas ha }^{-1}\right)\end{array}$} & \multicolumn{2}{|c|}{ Arranjo } & \multirow[t]{2}{*}{ Média } \\
\hline & Retângulo & Triângulo & \\
\hline & \multicolumn{3}{|c|}{ Massa do cacho $(\mathrm{kg})$} \\
\hline 1.333 & 29,81 & 30,78 & $30,30 \mathrm{a}$ \\
\hline 1.666 & 30,72 & 28,95 & $29,83 \mathrm{a}$ \\
\hline 2.222 & 28,73 & 29,47 & $29,10 \mathrm{a}$ \\
\hline 3.333 & 23,42 & 26,16 & $24,79 \mathrm{~b}$ \\
\hline \multirow[t]{2}{*}{ Média } & $28,17 \mathrm{~A}$ & $28,84 \mathrm{~A}$ & \\
\hline & \multicolumn{3}{|c|}{ Massa da ráquis $(\mathrm{kg})$} \\
\hline 1.333 & 2,47 & 2,24 & $2,36 \mathrm{a}$ \\
\hline 1.666 & 2,44 & 2,38 & $2,41 \mathrm{a}$ \\
\hline 2.222 & 2,44 & 2,37 & $2,40 \mathrm{a}$ \\
\hline 3.333 & 1,87 & 2,20 & $2,04 \mathrm{a}$ \\
\hline \multirow[t]{2}{*}{ Média } & $2,31 \mathrm{~A}$ & $2,30 \mathrm{~A}$ & \\
\hline & \multicolumn{3}{|c|}{ Massa de pencas por cacho $(\mathrm{kg})$} \\
\hline 1.333 & 27,34 & 28,54 & $27,94 a$ \\
\hline 1.666 & 28,28 & 26,57 & $27,42 \mathrm{a}$ \\
\hline 2.222 & 26,29 & 27,1 & $26,29 a$ \\
\hline 3.333 & 21,55 & 23,96 & $22,75 b$ \\
\hline \multirow[t]{2}{*}{ Média } & $25,87 \mathrm{~A}$ & $26,54 \mathrm{~A}$ & \\
\hline & \multicolumn{3}{|c|}{$\mathrm{MR} / \mathrm{MC}(\%)$} \\
\hline 1.333 & 8,28 & 7,28 & $7,78 \mathrm{a}$ \\
\hline 1.666 & 7,94 & 8,22 & $8,08 \mathrm{a}$ \\
\hline 2.222 & 8,50 & 8,04 & $8,27 \mathrm{a}$ \\
\hline 3.333 & 7,99 & 8,40 & $8,20 \mathrm{a}$ \\
\hline Média & $8,17 \mathrm{~A}$ & $7,99 \mathrm{~A}$ & \\
\hline
\end{tabular}

\begin{tabular}{|c|c|c|c|}
\hline \multirow{2}{*}{$\begin{array}{c}\text { Densidade } \\
\left(\text { plantas ha }^{-1}\right)\end{array}$} & \multicolumn{2}{|c|}{ Arranjo } & \multirow[t]{2}{*}{ Média } \\
\hline & Retângulo & Triângulo & \\
\hline & \multicolumn{3}{|c|}{ Frutos/cacho } \\
\hline 1.333 & 168 & 172 & $170 \mathrm{a}$ \\
\hline 1.666 & 174 & 164 & $169 a$ \\
\hline 2.222 & 165 & 181 & $173 a$ \\
\hline 3.333 & 156 & 154 & $155 \mathrm{a}$ \\
\hline \multirow[t]{2}{*}{ Média } & $166 \mathrm{~A}$ & $168 \mathrm{~A}$ & \\
\hline & \multicolumn{3}{|c|}{ Massa do fruto (g) } \\
\hline 1.333 & 162,73 & 165,90 & $164,70 \mathrm{a}$ \\
\hline 1.666 & 162,52 & 162,01 & $163,10 \mathrm{a}$ \\
\hline 2.222 & 159,33 & 149,72 & $154,53 \mathrm{ab}$ \\
\hline 3.333 & 138,14 & 155,58 & $146,86 \mathrm{~b}$ \\
\hline \multirow[t]{2}{*}{ Média } & $155,68 \mathrm{~A}$ & $158,30 \mathrm{~A}$ & \\
\hline & \multicolumn{3}{|c|}{ Pencas/cacho } \\
\hline 1.333 & 9,0 & 9,0 & $9,0 \mathrm{a}$ \\
\hline 1.666 & 9,0 & 8,5 & $8,7 a$ \\
\hline 2.222 & 8,5 & 9,0 & $8,7 \mathrm{a}$ \\
\hline 3.333 & 8,5 & 8,7 & $8,6 \mathrm{a}$ \\
\hline Média & $8,8 \mathrm{~A}$ & $8,8 \mathrm{~A}$ & \\
\hline
\end{tabular}

tição interplanta provocada pelo aumento do espaçamento, a ráquis tende a ter menor proporção em relação ao cacho, como consequiência da tendência de produção de frutos maiores. Esse fato pode ser comprovado pelos resultados obtidos em relação à massa de pencas por cacho e massa média do fruto, que aumentaram com a diminuição da densidade, embora o número de frutos e pencas por cacho não tenham apresentado diferenças significativas em função da densidade (Tabelas 1e 2).

A massa de pencas por cacho observada nas menores densidades ( 1.333 e 1.666 plantas ha ${ }^{-1}$ ) foi significativamente superior às verificadas na maior densidade (3.333 plantas ha-1), conforme Tabela 1 . O mesmo comportamento foi observado quanto à massa média do fruto (Tabela 2). Esses resultados também explicam a maior massa do cacho observada nas menores densidades. A diminuição da massa do

\section{TABELA 2. Número de frutos, massa do fruto e nú- mero de pencas da bananeira 'Nanicão'
em diferentes densidades e arranjos. mero de pencas da bananeira 'Nanicão'
em diferentes densidades e arranjos. Piracicaba, SP, 1997.}


fruto no plantio mais denso pode ser uma conseqüência do alto grau de sombreamento do bananal, o que diminui a capacidade fotossintética da planta e reduz o enchimento e desenvolvimento do fruto (Robinson \& Nel, 1988; Israeli et al., 1995).

Embora tenha diminuído a massa do cacho e do fruto, o aumento da densidade de 1.333 para 3.333 plantas ha-1 praticamente duplicou a produção de cachos em toneladas por hectare (Tabela 3), o que era esperado em virtude do maior número de plantas presentes.

A determinação da massa da ráquis permitiu estimar a produção por hectare desse material, de acordo com as densidades utilizadas (Tabela 3 ). A produção de ráquis aumentou com o incremento da densidade, tendo variado de $3,14 \mathrm{t} \mathrm{ha}^{-1}$, com 1.333 plantas ha-1 , a 6,80 t ha- ${ }^{-1}$, com 3.333 plantas ha ${ }^{-1}$.

TABELA 3. Produção estimada de cachos, frutos e ráquis da bananeira 'Nanicão' em diferentes densidades e arranjos. Piracicaba, SP, $1997^{1}$.

\begin{tabular}{|c|c|c|c|}
\hline \multirow{2}{*}{$\begin{array}{l}\text { Densidade } \\
\left(\text { plantas }^{-1}{ }^{-1}\right)\end{array}$} & \multicolumn{2}{|c|}{ Arranjo } & \multirow[t]{2}{*}{ Média } \\
\hline & Retângulo & Triângulo & \\
\hline & \multicolumn{3}{|c|}{ Produção de cachos $\left(\mathrm{t} \mathrm{ha}^{-1}\right)$} \\
\hline 1.333 & 39,75 & 41,04 & $40,39 c$ \\
\hline 1.666 & 50,84 & 48,23 & $49,53 \mathrm{bc}$ \\
\hline 2.222 & 63,84 & 65,48 & $64,66 a b$ \\
\hline 3.333 & 78,08 & 87,18 & $82,63 a$ \\
\hline \multirow[t]{2}{*}{ Média } & $58,13 \mathrm{~A}$ & $60,48 \mathrm{~A}$ & \\
\hline & \multicolumn{3}{|c|}{ Produção de frutos $\left(\mathrm{t} \mathrm{ha}^{-1}\right)$} \\
\hline 1.333 & 36,44 & 38,04 & $37,24 \mathrm{c}$ \\
\hline 1.666 & 47,11 & 44,26 & $45,68 \mathrm{bc}$ \\
\hline 2.222 & 58,40 & 60,21 & $59,30 \mathrm{ab}$ \\
\hline 3.333 & 71,88 & 80,79 & $75,83 \mathrm{a}$ \\
\hline \multirow[t]{2}{*}{ Média } & $53,56 \mathrm{~A}$ & $55,60 \mathrm{~A}$ & \\
\hline & \multicolumn{3}{|c|}{ Produção de ráquis $\left(\mathrm{t} \mathrm{ha}^{-1}\right)$} \\
\hline 1.333 & 3,29 & 3,00 & $3,14 \mathrm{c}$ \\
\hline 1.666 & 4,06 & 3,96 & $4,01 \mathrm{bc}$ \\
\hline 2.222 & 5,42 & 5,26 & $5,34 \mathrm{ab}$ \\
\hline 3.333 & 6,24 & 7,34 & $6,80 \mathrm{a}$ \\
\hline Média & $4,75 \mathrm{~A}$ & $4,66 \mathrm{~A}$ & \\
\hline
\end{tabular}

1 Em cada variável, médias seguidas de mesma letra, minúscula na coluna e maiúscula na linha, não diferem entre si pelo teste de Tukey a 5\% de probabilidade.
Com base na determinação da massa da ráquis estimou-se também a produção de frutos por hectare (Tabela 3). As diferenças estatísticas observadas em relação à produção estimada de cachos não foram alteradas, porém os valores absolutos foram proporcionalmente reduzidos, em função da porcentagem MR/MC.

Pelos resultados obtidos neste trabalho verificouse que a avaliação da massa da ráquis permite estimar o volume que pode ser produzido por hectare, desse material, em diferentes densidades. Além disso, pode-se estimar a produtividade de um bananal mais corretamente ao descontar-se a porcentagem de MR/MC. A duração do ciclo da bananeira, por sua vez, também deve ser levado em consideração na estimativa da produtividade de um bananal, cujo resultado é expresso em t ha-1 $\mathrm{ano}^{-1}$ (Robinson \& Nel, 1989).

A ráquis é um subproduto do cacho da bananeira que pode servir de matéria-prima para a fabricação de papel (Torres, 1981; Blanco Rojas, 1996). O seu aproveitamento torna-se mais importante em sistemas de produção em que as operações de despencamento, tratamento e embalagem são realizados em galpões, e não diretamente no campo. Quando a embalagem é realizada no bananal, a ráquis pode ser mantida sobre o terreno ou incorporada ao solo, como forma de repor parte dos nutrientes exportados na colheita (Vitti \& Ruggiero, 1984). Quando o cacho é transportado para o galpão de embalagem, a ráquis é pouco aproveitada após o despencamento. Nesse caso, poderia ser utilizada na fabricação de papel ou na alimentação animal.

Observa-se ainda que o adensamento de bananais promove maior volume de produção, por área, de cachos, ráquis e frutos, pela maior população de plantas. Entretanto, o maior número de plantas na área apresenta inconvenientes, como redução na massa do cacho e do fruto por planta, diminuindo a qualidade final do produto para mercado in natura. Isso torna o uso de altas densidades impraticável, quando se almeja o mercado externo. Por sua vez, o aumento da densidade de plantas pode ter aplicação prática na produção de matéria-prima para a indústria, na qual o tamanho do fruto não é tão importante, e na produção de ráquis, que poderia ser usada na fabricação de papel. 


\section{CONCLUSÕES}

1. A produção de ráquis de bananeiras 'Nanicão', submetidas a densidades de 1.333 a 3.333 plantas ha- ${ }^{-1}$, varia de 3,14 a $6,80 \mathrm{t} \mathrm{ha}^{-1}$

2. A porcentagem entre a massa da ráquis e a massa do cacho é de $8 \%$, independentemente da densidade e do arranjo de plantas.

3. O aumento na densidade de bananeiras 'Nanicão', de 1.333 para 3.333 plantas ha-1 ${ }^{-1}$, promove redução da massa do cacho e do fruto.

4. Há pouca interferência do arranjo (retângulo ou triângulo) sobre a produção da bananeira 'Nanicão' no terceiro ciclo.

\section{REFERÊNCIAS}

BLANCO ROJAS, M.L. Beneficiamento e polpação da ráquis da bananeira 'Nanicão' (Musa grupo AAA “Giant Cavendish"). Piracicaba : ESALQ, 1996. 150p. Dissertação de Mestrado.

DANIELLS, J.W.; O'FARREL, P.J.; CAMPBELL, S.J. The response of bananas to plant spacing in double rows in North Queensland. Queensland Journal of Agricultural and Animal Sciences, Brisbane, v.42, n.1, p.45-51, 1985.

FAO (Rome, Itália). Statistical databases. Disponível: FAO site (1999). URL: http://www.fao.org Consultado em 10 maio 1999.

GALLO, J.R.; BATAGLIA, O.C.; FURLANI, P.R.; HIROCE, R.; FURLANI, A.M.C.; RAMOS, M.T.B.; MOREIRA, R.S. Composição química inorgânica da bananeira (Musa acuminata cultivar Nanicão). Ciência e Cultura, São Paulo, v.24, n.1, p.70-79, 1972

GOMES, J.A.; NÓBREGA, A.C.; ANDERSEN, O. Densidade de plantio da bananeira cultivar Prata (grupo $\mathrm{AAB}$ ), na região produtora do Estado do Espírito Santo. In: CONGRESSO BRASILEIRO DE FRUTICULTURA, 7., Florianópolis, 1984. Anais. Florianópolis : Sociedade Brasileira de Fruticultura/ EMPASC, 1984. v.1, p.237-249.

ISRAELI, Y.; PLAUT, Z.; SCHWARTZ, A. Effect of shade on banana morphology, growth and production. Scientia Horticulturae, Amsterdam, v.62, n.1/2, p.45-56, 1995.

JANICK, J. A ciência da horticultura. Rio de Janeiro : Freitas Bastos, 1968. 485p.

LICHTEMBERG, L.A.; MALBURG, J.L.; HINZ, R.H. Effect of density on yield and cycle duration of Nanicão banana in Southern Brazil. Interamerican
Society for Tropical Horticulture Proceedings, Homestead, n.40, p.232-235, 1996.

MATTOS, J.R.; SIMÃO, S.; CAMPOS, H. Influência do espaçamento do peso dos cachos da bananeira. Solo. Piracicaba, v.62, n.2, p.51-60, 1970.

MOREIRA, R.S. Banana: teoria e prática de cultivo. Campinas : Fundação Cargill, 1987.335p.

PEREIRA, A.R. Competição intra-específica entre plantas cultivadas. Agronômico, Campinas, v.41, n.1, p.5-11, 1989.

PURSEGLOVE, J.W. Tropical crops: monocotyledons. New York: J. Willey, 1972. v.2, p.343-384.

ROBINSON, J.C.; NEL, D.J. Plant density studies with banana (cv. Williams) in a subtropical climate. I. Vegetative morphology, phenology and plantation microclimate. Journal of Horticultural Science, Ashford, v.63, n.2, p.303-313, 1988

ROBINSON, J.C.; NEL, D.J. Plant density studies with banana (cv. Williams) in a subtropical climate. II. Components of yield and seasonal distribution of yield. Journal of Horticultural Science, Ashford, v.64, n.2, p.211-222, 1989.

ROBINSON, J.C.; NEL, D.J.; BOWER, J.P. Plant density studies with banana (cv. Williams) in a subtropical climate. III. The influence of spatial arrangement. Journal of Horticultural Science, Ashford, v.64, n.4, p.513-519, 1989.

SANTOS, P.J. Estudo do comportamento da bananeira (Musa acuminata, Colla), cv. nanica em diferentes espaçamentos. Viçosa : UFV, 1977. 26p. Dissertação de Mestrado.

SIMMONDS, N.W. Bananas. 2.ed. London : Longmans, 1982. 512 p. (Tropical Agriculture Series).

SOTO BALLESTERO, M.S.; SOTO, E.; SOLÍS, P.; LÓPEZ, A. Siembra y operaciones de cultivo. In: SOTO BALLESTERO, M.S. Bananos: cultivo y comercialización. San José : Litografic e Imprenta, 1992. p.211-265.

TORRES, M. Propriedades fundamentales de la fibra del raquis del banano (Musa cavendishii). San José : Universidad de Costa Rica,1981. 31p.

VIDAL-TORRADO, P.; SPAROVEK, G. Mapa pedológico detalhado do Campus Luiz de Queiroz, Universidade de São Paulo. Piracicaba : ESALQ-Departamento de Ciência do Solo, 1993. Escala 1:10.000

VITTI, G.C.; RUGGIERO, C. Aproveitamento do engaço, coração e ráquis, como fonte de nutrientes. In: SIMPÓSIO BRASILEIRO SOBRE BANANICULTURA, 1., Jaboticabal, 1984. Anais Jaboticabal : UNESP/FUNEP, 1984. p.392-399. 